\title{
Du directeur au PDG (et inversement ?)
}

\section{Bryan Love}

\section{OpenEdition}

\section{Journals}

Édition électronique

URL : http://journals.openedition.org/ries/4193

DOI : $10.4000 /$ ries.4193

ISSN : 2261-4265

\section{Éditeur}

Centre international d'études pédagogiques

\section{Édition imprimée}

Date de publication : 1 décembre 1994

Pagination : 67-80

ISSN : 1254-4590

\section{Référence électronique}

Bryan Love, «Du directeur au PDG (et inversement?) », Revue internationale d'éducation de Sèvres [En ligne], 04 | 1994, mis en ligne le 17 avril 2015, consulté le 07 janvier 2020. URL : http:// journals.openedition.org/ries/4193; DOI : 10.4000/ries.4193

Ce document a été généré automatiquement le 7 janvier 2020

() Tous droits réservés 


\title{
Du directeur au PDG (et inversement?)
}

\author{
Bryan Love
}

1 Quiconque connaît bien l'histoire récente de l'Europe ne sera pas surpris d'apprendre que la Grande-Bretagne, avec toute son ambivalence quant à la façon de gouverner les États et tous ses paradoxes, exaspérants ou séduisants, est en réalité un État dépourvu d'enseignement national, ou tout du moins, d'un système d'enseignement national. Cependant, il est plus aisé de constater ce phénomène que de l'expliquer. Pour ce faire, je suis convaincu que nous devons nous appuyer sur une synthèse d'histoire, de culture et de politique et non sur des causes trop générales, une théorie pédagogique cohérente ou quelque projet ambitieux mais insaisissable. Et dans cette optique de simplicité, nous ne cherchons pas à savoir si la Grande-Bretagne est, en fait, un État unique ou un ensemble d'États.

\section{À propos du passé}

2 Ce qui passait pour de l'enseignement public, où des soi-disants public schools sont en fait l'apanage d'individus assez aisés pour éviter les écoles publiques, était, jusqu'à une période récente, plus précisément décrit comme un enseignement local ou régional. Les Local Education Authorities (LEA) furent mises en place en Angleterre et au Pays de Galles en 1944 afin d'organiser, diriger, financer (avec l'aide du gouvernement national) et superviser l'enseignement dans leurs zones respectives. Fréquemment, mais pas systématiquement, attenantes aux County, Borough ou aux City Concils, les LEA étaient assez proches de systèmes éducatifs restreints et nationaux existant ailleurs. Le système écossais était légèrement différent, mais pas au point d'affecter la thèse de cet article. Afin d'être le plus clair possible, je me limiterai à des remarques sur l'Angleterre. Ceci ne fait que donner plus de poids à mes arguments.

Dans les LEA anglaises, des conseillers élus formaient des comités d'enseignement et nommaient des directeurs d'enseignement professionnels. Les inspecteurs locaux ou les services consultatifs qui veillaient à ce que la politique de la LEA en matière de 
programmes soit dûment exécutée, ainsi qu'un corps financier et une équipe administrative présentaient leurs rapports à chaque directeur de la LEA. À eux tous, ils étaient responsables de l'enseignement dans leurs zones respectives. Les chefs d'établissement dirigeaient leurs écoles au nom de la LEA, présentaient des rapports de pure formalité à leurs conseils d'établissement et jouissaient d'une certaine liberté concernant l'utilisation de budgets limités destinés à servir le programme scolaire (il s'agissait principalement de fournir aux élèves des manuels gratuits). Cette liberté s'étendait aussi à d'autres domaines de moindre importance. Les salaires des enseignants et l'entretien des bâtiments, qui représentaient de loin les dépenses les plus élevées, étaient gérés par la LEA. Pourvoir les établissements en personnel et ouvrir un nombre suffisant de classes dans ces dernières était aussi de leur ressort.

4 Les directives nationales étaient rares. La seule matière obligatoire était l'éducation religieuse. Les LEA se sont développées chacune à leur manière, ce qui fait naître entre elles des différences frappantes. Ainsi, certaines sont restées fidèles à la sélection, mais la plupart ont été gagnées par la comprehensive revolution. Mais il existait mille et une façons d'interpréter ce nouveau type d'enseignement. Cette diversité endémique de l'enseignement en Grande-Bretagne se manifestait de plusieurs façons: ainsi, l'âge d'entrée dans le secondaire était variable, le principe de l'enseignement comprehensive était interprété de différentes façons et diverses solutions étaient apportées au problème du mode d'organisation de l'enseignement pour adultes.

De façon générale, je pense que les enseignants et la population dans son ensemble se sont bien accommodés de cet état de choses. Si irritation il y avait, elle tendait à s'exprimer à travers des critiques à l'encontre du gouvernement central, car les échelles des salaires étaient négociées au niveau national et le niveau des dépenses locales était, en pratique, fonction de la politique gouvernementale. Mais, en politique, il existe une force qui entraîne inexorablement l'enseignement dans l'arène politique des partis, et particulièrement lorsqu'on estime nécessaire de faire diversion à l'insatisfaction politique ou économique; et cela quelle que soit la couleur du gouvernement.

6 Mais il était dit que tout cela était destiné à changer, principalement sur l'ordre de politiques ambitieux ayant le sens de leur réputation si ce n'est de leur carrière. A l'exception de l'aménagement de notre Programme national, tout ce qui a été dit et fait cherchait moins à unifier nos systèmes distincts au nom de besoins nationaux qu'à les détruire. Il semblait que les systèmes, de tout type et de toute taille, avaient fait leur temps et que les unités individuelles étaient à la mode, en dépit de la tendance aux objectifs nationaux, et en dépit du fait que, logiquement, l'étape ultérieure aurait pu être un système national unique. En un mot, on ne jurait que par le petit.

7 Par parenthèse, et sans m'attarder davantage sur ce point, je dois avouer que j'aurais regretté une évolution vers un système national, tout autant que je regrette cette attaque menée contre nos systèmes locaux ou régionaux. Mais j'aurais respecté une telle évolution car elle aurait été sensée. L'histoire que je dois raconter à présent est, elle, pimentée d'illogismes et davantage motivée par l'opportunisme politique que par l'optique pédagogique. Je n'ai aucun plaisir à la raconter. 


\section{À propos du marché de l'enseignement}

8 Paradoxalement, le gouvernement conservateur de ces dernières années a été tout sauf conservateur. Ce qui est intéressant, c'est que des générations de nobles Tories ont cédé la place à une classe moyenne de self-made men dans les enjeux du pouvoir. Il suffit de regarder les origines sociales de ceux qui nous gouvernent aujourd'hui et de les comparer à celles des ministres conservateurs qui ont précédé Sir Edward Health et Lady Thatcher. Imperceptiblement, en l'espace de peu d'années, le cri de guerre s'est transformé ; les obligations de l'aristocratie terrienne se sont effacées devant la valeur de l'argent, sous-tendue par une foi, au départ inexprimée, dans le marché, une conviction que le monde des affaires, et l'entreprise privée en particulier, apportaient les réponses aux questions soulevées par les services publics et les industries de service nationalisées. Il ne fallut que quelque temps au marché pour acquérir et affirmer une pseudo-moralité au sein des services publics, avec la privatisation de certains d'entre eux comme l'eau, le gaz et les télécommunications. D'autres, comme les chemins de fer et les services postaux sont actuellement en phase préparatoire. Et même dans des domaines aussi sacrés que la santé et l'éducation, des "marchés internes » se créent rapidement. Aujourd'hui, même notre fonction publique, l'infrastructure routière et les prisons sont menacées. Cette tendance était peut-être à prévoir, vu le nouveau profil social des dirigeants Tory que je ne suis pas en mesure d'expliquer.

9 Les marchés ont besoin de clients bien disposés, de produits et de stratégies commerciales là où les services publics ont pour principe de satisfaire de façon plus ou moins altruiste les besoins perçus. Mais percevoir un besoin et formuler un souhait sont deux choses bien différentes. Ainsi les marchés sont-ils conçus pour fournir à nos clients ce qu'ils veulent et les services publics cherchent à leur donner ce que nous pensons qu'ils devraient vouloir! Tout cela est en fait moins draconien qu'il n'y parait : le contrôle et la responsabilité politiques au niveau local ou national garantissent, dans la pratique et dans une large mesure, la " protection du consommateur ». Les marchés changent rapidement, par conséquent, leur optique est essentiellement de court terme. Les services publics en général, et les systèmes éducatifs en particulier, ont une vue à plus long terme. Ces observations ne sont nulle part mieux mises en relief que dans une étude concernant ce qui est arrivé à l'inspection centrale ${ }^{1}$.

10 Avant l'avènement du nouvel ordre, l'inspection nationale était une section indépendante de la fonction publique, et elle avait libre accès aux écoles. Elle organisait aussi des cours pour enseignants. Si son standing professionnel était généralement élevé, ses avis étaient parfois mal accueillis par les hommes politiques. Peu à peu s'est développée au sein du gouvernement l'idée que le HMI lui-même était une force de réaction, faisait partie de l'establishment et qu'il devait être affaibli, si ce n'est éradiqué. Ainsi, dans une de ses réformes les plus spectaculaires, le gouvernement a-t-il allégé le HMI et créé l'OFSTED².

11 Aujourd'hui, l'OFSTED supervise les inspections privatisées de chaque école tous les quatre ans, en théorie du moins. En pratique, de plus en plus de doutes apparaissent quant à la capacité de l'organisme à instaurer et maintenir ce rythme. Des équipes individuelles d'inspecteurs, agissant théoriquement comme des entreprises commerciales distinctes, et contenant chacune un inspecteur qui n'a jamais travaillé dans le domaine éducatif et dont la seule qualification en matière d'inspection repose sur un stage d'une semaine, proposent leurs services. L'équipe gagnante sélectionnée et 
payée par l'OFSTED descend dans une école et y reste environ une semaine, écrit son rapport, le publie à l'intention de tous et disparaît. À la différence de l'HMI, elle n'a aucun moyen d'aider l'école à se développer, et à la différence des inspections des anciennes LEA, elle n'a pas pour devoir d'aider l'école à s'améliorer. «Inspecter pour améliorer » est un slogan politique très en vogue, mais « humilier pour faire changer » semble être l'ordre du jour dissimulé.

En termes simples, la philosophie du marché et la culture d'entreprise du système politique britannique semblent avoir été liée non seulement à un rejet des systèmes, mais aussi à une défiance à l'égard des experts. Ceux d'entre nous qui vivent ici ont pris l'habitude d'entendre ce mot prononcé avec un mépris à peine déguisé. On se souviendra surtout de l'ancien ministre John Patten pour avoir donné à un membre du monde scolaire hautement respecté l'étiquette infâme de "cinglé ». Laisser entendre que la compétence est dangereuse est bien évidemment ridicule, mais il est néanmoins vrai que sa force politique est réelle dans certains domaines. Il fallait rendre l'HMI impuissant en invoquant la nécessité de discréditer ce qui était dépeint comme subversif. Dans le même temps, donner officiellement le pouvoir à ceux qui n'avaient pas été corrompus par le monde de l'éducation, à savoir les inspecteurs "hors du sérail ", était rien moins que magistral. Parallèlement, vaincre l'inertie des services publics en injectant une bonne dose de capitalisme et en opposant les inspecteurs les uns aux autres compensait en popularité un manque de rigueur intellectuelle évident. C'était du moins la chose espérée. Seul le temps dira dans quelle mesure ce radicalisme de la droite sera populaire ou non. Il fait certes preuve de simplicité, mais certainement pas de profondeur.

Mais le lien entre le dénigrement des experts et la foi dans le marché est véritablement complexe à démêler. Les experts, nous dit-on, se nourrissent d'autres experts. La compétence devient son propre objectif dans un monde intellectuel ou universitaire replié sur lui-même, et qui se perpétue dans une autosatisfaction totale. Ce monde s'oppose au monde "réel », avec tout ce que cela veut signifier, c'est-à-dire gagner sa vie en étant meilleur (voilà un mot intéressant) que son voisin, qui n'est autre que son concurrent, et en répondant exclusivement aux besoins de son client. Ce nouveau monde, le monde réel, génère néanmoins son propre type d'expert, mais il l'appelle manager. Les managers, motivés par l'argent, qui leur confère mérite et prestige, ne sont considérés comme experts que dans le domaine du management et, de ce fait, conservent un certain degré d'innocence. Ainsi, le manager prospère, motivé par la réussite financière, passe, disons par exemple, de l'industrie ferroviaire (dont les passagers sont aujourd'hui appelés "clients ») à la fonction publique ou, pour citer un exemple «réel», d'une compagnie pétrolière multinationale à un bureau d'aménagement des programmes scolaires. Il est facile de trouver d'autres exemples. Sir Ron Dearing, appelé par John Patten pour supprimer en partie la législation chaotique des programmes mis en place par un de ses prédécesseurs conservateurs, a été recruté dans le monde des grandes entreprises, comme l'a été un président de ce qui était alors le Conseil des examens et de l'évaluation (School's Examination and Assessment Council). 


\section{À propos des LEA} traditionnellement très apprécié dans ce pays et par ce gouvernement, semble-t-il. Certains "pédagogues amateurs", tels que ceux que j'ai déjà mentionnés, sont effectivement rétribués pour leur action. Mais, à un niveau différent, il en existe de nombreux pour qui ce n'est pas le cas. A travers le pays, on a donné de nouveaux pouvoirs et responsabilités aux conseils d'établissement, et ceci aux dépens de l'autonomie d'antan des LEA et des chefs d'établissement. Il est clair que le modèle utilisé est le conseil d'administration industriel et commercial, avec un chef perçu comme un PDG.

15 Mais le monde de l'enseignement a continué à être attaqué sur d'autres fronts significatifs. Poursuivons avec les LEA. On peut facilement imaginer la réunion stratégique des généraux à la veille de la bataille : «Comment pouvons-nous renverser leur position monopolistique en introduisant la concurrence et, par conséquent, l'efficacité du marché ? Bon, là où il n'existe pas de concurrent évident, il est nécessaire d'en inventer un. Cela devrait faire l'affaire. Cela rendra les experts moins suffisants et, du même coup, plus concernés par les enjeux vitaux dès l'instant qu'ils dirigent un marché. Mettez le feu aux poudres et gardez vos distances. Mais, au cas où cela ne produirait pas l'effet désiré, trouvons des façons de rendre les LEA plus compétitives entre elles.»

16 La plupart d'entre nous peuvent se souvenir d'une époque à laquelle nous ne savions pas ce que coûtait l'administration de nos écoles. Certains pensent que ce n'était pas si mal, que c'était une marque de civilisation. Après tout, nous étions des enseignants, pas des hommes d'affaires. Nous n'étions pas supérieurs aux hommes d'affaires, simplement différents. Ce qui nous avait motivés, c'était la satisfaction de faire un métier du mieux que nous le pouvions, mais ce n'était certainement pas le salaire perçu ! La vocation était un terme qui signifiait plus pour nous que tout autre. En tant que chefs d'établissement, nous passions notre temps à essayer d'améliorer la qualité de l'expérience scolaire de nos élèves. Nous observions des cours, la plupart d'entre nous continuaient à en donner au moins quelques-uns. Nous parlions aux gens, planifions, évaluions, apprenions, passions du temps à résoudre les problèmes d'individus et de groupes, nous réfléchissions beaucoup et travaillions dur. Nous avions le temps d'être des dirigeants, et beaucoup d'entre nous considéraient cette activité comme plus noble que le management.

Nous ne savions pas ce que coûtait l'administration de nos écoles parce que nous n'avions pas besoin de le savoir ; ce qui était en quelque sorte un luxe, mais un luxe que nous pouvions nous offrir puisque, comme nous l'avons vu, les LEA, avec leurs économies d'échelle, employaient des comptables et des planificateurs financiers de façon centralisée (mais pas autant que nous ne le faisons aujourd'hui individuellement) pour faire le travail à notre place. Les LEA nous disaient combien d'enseignants nous pouvions employer, combien nous pouvions les payer, et elles nous donnaient l'argent pour les trouver et les garder. Et si nous avions un besoin particulier, par exemple conserver les cours de latin une année où trop peu d'élèves avaient choisi de l'étudier, ou bien entreprendre la réparation d'un bâtiment de façon imprévue, ou encore chauffer nos classes lors d'un hiver rigoureux, la LEA résolvait le problème pour nous. Tout du moins, la plupart des LEA. Le HMI avait l'œil rivé sur celles qui ne résolvaient 
pas ce genre de problèmes, et il est vrai que certaines y parvenaient moins bien que d'autres. Il est également vrai que certaines, très peu en fait, promulguaient des politiques de programmes assez ésotériques ou radicales. C'est pourquoi le gouvernement, en prétendant justifier son action par l'existence de cette brebis galeuse, a littéralement trouvé une formule pour faire maigrir la bête. Pour commencer, le financement centralisé des LEA a été décrit comme vicié par essence. L'argent était gaspillé, a-t-on dit, à aider des écoles en situation d'échec. On a le sentiment, à considérer toutes ces années durant lesquelles le parti conservateur a critiqué les écoles publiques que, selon lui, nous étions tous en situation d'échec. Mais, à ce stade du débat, le gouvernement a émis des critiques plus sélectives et a voulu que les LEA se différencient également. Puis il a poursuivi en déclarant que dépenser plus pour réparer le toit de l'école A plutôt que celui de l'école B était profondément injuste. Ensuite, à cette injustice, ont été amalgamés un manque d'efficacité dans la dépense des fonds publics ainsi qu'un manque de transparence, expression ô combien intéressante en matière de dépenses publiques. En ayant recours à ce simple expédient, le gouvernement a transformé le débat habituel sur la qualité de l'enseignement, axé sur les fonds nationaux, le gouvernement central et les hommes politiques nationaux, en un débat sur les dépenses locales, axé cette fois sur les LEA, les politiques locaux, les conseils d'établissement et les managers. Ces derniers incluaient bien sûr les chefs d'établissement. En bref, le gouvernement est passé de «Combien? » à «Comment?». Souvenons-nous également que le marché est essentiellement une affaire de dépense sélective. Ainsi, la question de la qualité s'est trouvée davantage liée à la pertinence des dépenses qu'à celle des recettes. Un pas de plus, et le rapport qualité-prix sera considéré comme le pivot central du débat sur l'enseignement.

La manœuvre suivante du gouvernement a été d'exiger que chaque LEA publie l'ensemble de son budget, réduise la part de ce budget attribuée à ses services centraux (ce qui a mis de nombreux experts au chômage) et répartisse la somme restante parmi ses écoles selon une formule qui devait être fort simple.

Le gouvernement était très fier de ses réalisations. Il se sentait en mesure d'affirmer qu'il avait libéré les écoles du contrôle politique - en oubliant, de façon fort opportune, qu'il s'était contenté de remplacer une forme de contrôle politique par une autre, qu'il avait fermement placé le pouvoir entre les mains des parents (transformés en clients), obligé les écoles à répondre de leurs actes devant leurs communautés et rendu la gestion des écoles plus motivée et plus efficace.

\section{À propos de la concurrence scolaire}

20 Les personnes enthousiasmées par tout cela, y compris un nombre important de chefs d'établissement, éprouvent un sentiment de libération mais, comme sur tous les marchés, même la libération a un prix. Le sentiment de libération de ceux qui se délectent de leur autonomie toute neuve existe au prix du sacrifice du système ou, si vous préférez, des systèmes. Et le système n'existait que pour une seule raison, garantir à chaque jeune un établissement scolaire adapté à ses besoins. Tel était l'objectif de l'acte promulgué en 1944. Pour réaliser ce but, les LEA ont planifié le nombre et le type d'établissements scolaires dans chaque zone. Les parents qui, à l'époque, n'étaient pas des clients, se voyaient attribuer un établissement scolaire pour leurs enfants. Nous 
pouvons nous interroger sur l'adéquation et la qualité de ce dernier, mais on peut difficilement contester le principe en lui-même. système local, des plans étaient élaborés, soit pour hâter la fin du malade et abréger ses souffrances, soit pour le soigner. Les lecteurs innocents croiront peut-être difficilement que ceci est aujourd'hui quasiment impossible. L'élève de cette école tombée en désaffection se retrouve dans un contexte de déclin financier, de chances toujours plus réduites de terminer son cursus scolaire, il est entouré d'une équipe démoralisée qui tremble pour son emploi et, très certainement, de bâtiments qui s'effritent tout autant que le moral des élèves. Un tel climat peut persister durant une génération entière d'élèves car le dénouement, inévitable, est long à venir. Aucune force du marché ne peut sauver une telle situation. La théorie selon laquelle ces pressions entraîneront d'elles-mêmes une amélioration est par trop naïve. Elle traduit une incompréhension totale du psychisme des enseignants et un manque total d'égards pour la situation critique dans laquelle se trouvent les élèves. Les écoles ne sont pas des entreprises, c'est sûrement là un des arguments les plus faciles à défendre.

L'autre aberration qui nous importe ici est de croire que la nouvelle formule de financement est en quelque sorte plus juste que l'ancienne méthode qui était fonction des besoins. On confond équité et changement. Nous avons vu que cette formule dépend principalement du nombre d'élèves qu'une école peut attirer. Par conséquent, les dépenses importantes autres que celles en rapport avec les élèves passent 
inaperçues. Le meilleur exemple à fournir est celui des bâtiments. Une école de 1000 élèves peut très bien posséder un ou deux bâtiments de briques, vastes et solidement construits, avec des toits en pente. Cette école devra dépenser bien moins en entretien et autres frais d'exploitation que sa concurrente de taille similaire, mais composée d'un ensemble de petites constructions bon marché abritées par des toits plats. Ainsi, la première école aura plus d'argent à dépenser pour les enseignants et les manuels. C'est là un problème bien réel de cette nouvelle organisation, mais une vérité très bien cachée aussi.

Voilà pour l'instant ce qui concerne la première étape de la création d'un marché à l'intérieur des LEA. Mais nous aurons d'ici peu à revenir sur ce thème, car il y a encore d'autres points à signaler à ce propos. Cependant, nous n'en avons pas terminé avec l'étude de la concurrence entre écoles. Revenons donc à la réunion stratégique de nos généraux, au moment précis où nous avons laissé ces héros en train de réfléchir à la façon de créer une concurrence aux LEA.

\section{À propos des établissements autonomes}

27 Les écoles financées par la nouvelle formule prennent elles-mêmes leurs décisions. Là où elles auraient pu autrefois critiquer leur LEA lorsqu'elles considéraient qu'elles manquaient d'enseignants, elles sont maintenant seules responsables. Même s'il arrive que les revenus ne suffisent pas à couvrir les dépenses, cet argument n'est pas toujours défendable. Certaines écoles n'ont été que trop enclines à souhaiter des sources de financement supplémentaires. Le gouvernement y a été attentif. C'est le gouvernement central qui décide en grande partie combien chaque LEA peut consacrer à son budget d'enseignement. Mais n'étant pas entièrement seul en cause, il en a toujours profité pour faire dévier les critiques portant sur un financement inadéquat. Il faut dire que les gouvernements ont parfois eu de bonnes raisons de le faire. Mais souvent, rien ne les y autorisait.

28 Le gouvernement conservateur a cherché à tirer profit du financement inadéquat des écoles, quelles qu'en soient les causes, et à porter son attaque idéologique à l'encontre des LEA en permettant aux écoles de "se soustraire " au contrôle de ces dernières. Ainsi, sur un simple vote des parents (où les enseignants ne peuvent faire entendre leur voix), une école peut très bien abandonner sa LEA pour devenir grant maintained ${ }^{3}$. Au début, d'énormes avantages financiers étaient concédés aux écoles qui choisissaient de se soustraire au contrôle des LEA et bénéficiaient ainsi de budgets considérablement plus élevés. Mais aujourd'hui, les gains financiers sont moindres, quoique néanmoins importants. Les subventions sont distribuées par un soi-disant conseil de financement, lui-même une extension du gouvernement national; cependant ce sont les LEA qui doivent fournir la majeure partie des fonds! Les conséquences pour les LEA sont évidentes. Ce sont les écoles relevant des LEA qui font les frais des écoles subventionnées. Elles disposent d'un éventail réduit de services de la LEA, pâtissent de la réduction du budget de celle-ci, et sont forcées d'entrer dans un environnement compétitif quand d'autres facteurs locaux et accords informels auraient pu les aider à y échapper.

29 L'initiative décrite ci-dessus a été un échec embarrassant pour le gouvernement, car très peu d'écoles ont choisi de se retirer. Peut-être les écoles ont-elles vu ce retrait comme une façon d'avoir des liens plus étroits avec le gouvernement central, mais sans 
les avantages que confère l'appartenance à un système national proprement dit. Parmi les écoles devenues grant maintained, on trouve des candidats à la fermeture pour cause de diminutions d'effectifs. Mais on doit reconnaître que certaines écoles très performantes (en termes d'économie de marché) ont mordu à l'hameçon. Quelle qu'en soit la raison, les zones touchées par le virus du subventionnement ont toutes manifesté le même symptôme, à savoir une capacité à planifier singulièrement réduite. Il suffit à une école menacée de fermeture par une LEA de se retirer pour semer la confusion dans le budget et la gestion prévisionnelle locale de l'éducation. Le gouvernement a mis en œuvre d'autres stratagèmes pour mettre fin à la confortable harmonie des LEA. Ainsi les City Technology Colleges furent-ils curieusement conçus pour s'assurer le financement (et une grande part du contrôle de l'enseignement) du monde des affaires et de l'industrie, financement qui serait axé sur une "école aimant " destinée à injecter davantage de concurrence dans l'enseignement. Cette initiative a rencontré encore moins de succès que celle des écoles grant maintained.

Les réactions des LEA face à l'étendue et l'extraordinaire rapidité de ces changements ont été fort ingénieuses. Confrontées à des fonds de plus en plus restreints mais aussi, ce qui est quelque peu illogique, à une obligation légale de continuer à exister et ainsi fournir certains services, y compris ce que les supermarchés appelleraient des articles pilotes, elles se sont restructurées de façon très efficace et se sont mises à courir après la clientèle. Avant tout, elles ont donné un prix à leurs différents services et espéré que les écoles, y compris les grant maintained, et celles dépendant d'autres LEA, achèteraient ces services. Dans l'ensemble, c'est ce qui s'est produit car, en pratique, les écoles ont découvert dans leur autonomie accrue un pouvoir caché, à savoir subvertir en partie la culture de marché et conserver autant que possible les avantages d'une coopération avec d'autres écoles et la LEA.

\section{À propos du marché interne}

31 Néanmoins, le gouvernement peut prétendre avoir créé un marché interne dans l'enseignement public. Nous avons vu comment il se présente aux yeux d'un groupe de clients, les parents, qui en théorie peuvent choisir le type et l'emplacement de leur école. Mais il existe un autre groupe de clients : les écoles elles-mêmes. Qu'est-il pour elles? En un mot, tout cela leur semble être un leurre. Elles dépensent habituellement environ $85 \%$ de leur budget en dépenses de personnel, ce qui laisse bien peu lorsque s'y ajoutent d'autres frais plus ou moins fixes. Ainsi, la plupart des écoles se contentent de recycler l'argent restant dans les LEA, en achetant les mêmes services aux mêmes personnes qui les leur auraient de toute façon procurés si rien n'avait changé. Aujourd'hui bien sûr, tout ce processus est plus bureaucratique que par le passé, puisqu'il implique des décisions budgétaires, des factures et tout l'attirail du monde des affaires.

Cependant, il est vrai que certaines écoles achètent certains services auprès d'autres LEA que les leurs, ou même auprès de "consultants " indépendants. Cette espèce nouvelle inonde chaque jour un peu plus nos bureaux de brochures glacées, qui, je suppose, doivent être payées d'une façon ou d'une autre, et elle est un des signes de la culture d'entreprise existant au sein de l'enseignement. En effet, nombre d'entre eux sont consultants en marketing ou comptables. De façon générale cependant, les écoles 
ont réussi à continuer comme par le passé. Mais ce qui est triste, c'est que l'effort déployé pour ce faire montre bien que le management l'emporte sur la direction.

Mais il existe une différence notable qui a des répercussions sur la responsabilité des écoles. Il fut une époque où une LEA décidait d'inspecter ses écoles, parfois à la requête des chefs d'établissement, mais pas toujours, et contrôlait tout changement jugé nécessaire. A présent, il est plus normal que les LEA offrent des services consultatifs, payés par les écoles, et compensent le manque de moyens en faisant des inspections d'écoles d'autres LEA et liées par contrat à l'OFSTED. Ainsi, des équipes d'inspection des LEA sillonnent le pays et sortent de leurs zones d'influence pour inspecter les écoles d'autres LEA (mais elles n'ont pas le droit d'inspecter des écoles qu'elles "connaissent»). Ceci semble produire les trois résultats suivants: manque de disponibilité dans leur propre zone auprès des écoles qui dépendent d'elles, difficulté à distinguer les services publics des services privés, et revenu accru pour nos chemins de fer bientôt privatisés. Si cette tendance se poursuit, il ne fait aucun doute que l'on ira de plus en plus chercher conseil au-delà de l'équipe locale en matière de programmes scolaires. Et pourquoi cette tendance devrait-elle se limiter aux seuls programmes?

Donc voici le marché de l'enseignement et la culture d'entreprise tels qu'en euxmêmes. J'ai laissé de nombreux détails de côté, tels que la Charte des parents imprimée sur papier glacé et distribuée dans chaque foyer, ce qui fut extrêmement coûteux, ou la publicité que j'ai entendue à la radio cet été qui présentait les places de telle université comme s'il s'agissait d'une poudre à laver, ou encore les publicités publiées à grands frais par le gouvernement dans la presse nationale pour vanter les mérites d'un test chez les élèves de quatorze ans ou le fait que les commissions d'examens elles-mêmes sont à présent des entreprises parmi lesquelles les écoles peuvent choisir de façon à faire la meilleure affaire.

Cette histoire ne serait pas complète sans un dernier chapitre, et le dénouement défie toute logique. En 1944, année où les LEA furent mises en place, seule l'éducation religieuse était obligatoire. Aujourd'hui nous avons le Programme national. C'est ainsi qu'il se nomme, mais il serait plus exact de dire le Programme des Deux Nations, car il ne s'applique pas au secteur privé. Ce Programme national, dont la mauvaise gestion constituerait à elle seule le sujet d'un article divertissant si elle n'entachait pas les chances d'une génération entière d'élèves, détermine le contenu pédagogique et l'évaluation de pratiquement tout ce que nous faisons aujourd'hui. Ainsi, et d'un seul coup, le gouvernement a-t-il décentralisé encore plus nos structures déjà décentralisées, réduit à néant nombre des caractéristiques d'un système, et promulgué des slogans tels que "choix et diversité ", mais paradoxalement, il nous a quasiment ôté toute possibilité de choisir parmi plusieurs programmes. À cet égard, la centralisation est bien plus importante aujourd'hui qu'hier, l'homogénéité aussi. Il existe bien sûr des arguments respectables en faveur de la centralisation, mais ils sont peu compatibles avec les autres « réformes ».

\section{À propos du métier de chef d'établissement}

En dépit du tableau que j'ai dépeint, je pense que la plupart d'entre eux sont des chefs d'établissement et non des enseignants. Mais je dois admettre que moi aussi je trouve assez stimulant d'être responsable d'un budget de trois millions de livres, de défendre mon point de vue auprès des membres du conseil d'établissement, de trouver le moyen 
de faire ces petites choses que la bureaucratie aurait rendues impossibles et d'avoir plus de pouvoir pour prendre des décisions considérées comme importantes. Mais à mon avis, tous ces attraits ont davantage à voir avec notre psychologie qu'avec notre éthique ou nos valeurs. Nous faisons contre mauvaise fortune bon cœur et trouvons des moyens pour que notre métier demeure agréable. Tels Estragon et Vladimir, nous jouons à faire passer le temps en attendant Godot. Mais, comme eux, nous nous retrouvons dans le jeu de quelqu'un d'autre, que l'on veuille jouer ou non. Aussi, je dois mentionner l'existence d'un nombre grandissant de supporters de certaines de ces réformes ; ce sont ceux qui sont contents de jouer, principalement les gagnants, mais je ne peux pas parler en leur nom.

Philosophiquement parlant, j'observe sans plaisir toutes les réflexions nébuleuses qui entourent des thèmes tels que : système et marché, centralisation et décentralisation, compétence et amateurisme (après tout, l'ignorance est plus exactement l'antithèse de la compétence), utilisation optimale du temps des professionnels, et enfin meilleures méthodes de financement des écoles. Je constate avec déception que progressivement mon travail est moins affaire de rapports humains que de chiffres. Professionnellement parlant, je me sens un peu coupable quand je réalise dans quelle mesure quelqu'un qui exerce mon métier a le pouvoir de dire la vérité et ne le fait pourtant pas. La cause en est à chercher dans l'industrie d'emballage! Achèteriez-vous un bocal de café dont l'étiquette déplore les conditions dans lesquelles ce bocal a été produit? Enverriez-vous vos enfants dans une école dont le chef d'établissement est un rouspéteur notoire? L'emballage a triomphé sur le contenu. Nous publions des prospectus sur papier glacé avec l'argent que nous devrions dépenser en manuels. Nous cherchons comment présenter au mieux nos résultats d'examens ou nos rapports d'inspection et nous affichons toujours un sourire de circonstance. Certaines écoles doivent même avoir recours à des techniques encore plus voyantes pour copier les pratiques commerciales. Je n'ai pas encore entendu parler d'une école qui offre des T-shirts gratuits aux parents qui inscrivent leurs enfants, mais ce jour viendra sûrement. Si vous avez des doutes, réfléchissez à ce projet de nouveaux programmes émis par le SCAA (School's Curriculum and Assessment Agency), et que j'ai récemment reçus dans un sac en plastique recouvert d'un logo, du type de ceux que les vendeurs vous mettent dans la main au Salon et de l'Automobile.

38 Et pourtant je demeure optimiste, et ceci pour deux raisons. La première est encore une fois politique. Comme je l'ai déjà suggéré, je pense qu'il y aura un choc en retour. J’ai de nombreuses raisons de le croire, trop nombreuses pour être décrites ici. Je vais donc me limiter à deux exemples d'ineptie politique.

Le pouvoir conféré aux membres des conseils d'établissement s'inscrivait, je crois, dans le mouvement lancé à l'encontre de la compétence professionnelle. Mais peu de ces membres restent des amateurs, si ce n'est dans la mesure où ils ne perçoivent pas de salaire. Ils ne sont assurément pas ignorants. Ils doivent simplement exploiter leurs connaissances pour survivre. La plupart d'entre eux deviennent les alliés indéfectibles des enseignants, les admirateurs de leur travail, supporters de leur cause et, dans certains cas, ils se font bruyamment les avocats de leur mérite. Les membres de nos conseils d'établissement, et je parle ici pour l'ensemble des écoles, sont devenus des visiteurs bienvenus dont les avis sont très appréciés. Ils prêcheront, tout comme les enseignants, la bonne parole en temps opportun. Les enseignants et les membres des conseils d'établissement réunis doivent constituer une part significative de l'électorat. 
40 On a encouragé les parents à penser qu'ils étaient des consommateurs. Pourtant, comme je l'ai montré, le marché ne peut pas satisfaire toutes leurs exigences de consommation. Un consommateur mécontent est plus dangereux pour un homme politique que l'utilisateur d'un service qui ne s'attend pas à pouvoir choisir. Voilà un exemple de ce que j'appelle l'effet Gorbatchev et je note avec satisfaction que notre presse nationale s'exprime de plus en plus fort sur ce sujet. Les groupes de parents sont, eux aussi, de plus en plus conscients que tout n'est pas conforme à ce qui leur est dit.

41 Je note aussi que certains parents refusent tous ces changements. Ils se souviennent de leur scolarité avec émotion et ne demandent rien d'autre que de retrouver la confiance qu'eux et leurs parents plaçaient dans l'enseignement lorsqu'il était dirigé sans états d'âme par des experts. Ils font un parallèle avec leur médecin ou leur dentiste en qui ils ont confiance pour toutes sortes de raisons. Un jour, tous ces gens diront ce qu'ils pensent.

42 La deuxième raison pour laquelle je reste optimiste est que mon métier est toujours le plus beau du monde. Même si les politiques souhaitent qu'il en soit autrement pour quelque raison que ce soit, je suis toujours entouré d'êtres humains autant que de bilans. Il reste à éduquer et aider les élèves. Les enseignants, qui conservent leur fierté et leur bonne humeur face aux diatribes gouvernementales, sont toujours des gens agréables à fréquenter. Les parents continuent à compter sur moi lorsqu'ils ont besoin d'aide. Les membres du conseil d'établissement m'apportent une aide véritable et une mine de bonnes idées; et mes collègues de la LEA, du moins ceux qui restent, sont de véritables alliés professionnels. Toutes ces personnes me permettent de poursuivre. Les chiffres peuvent attendre.

43 Ma tâche était de décrire et d'évaluer, pas de recommander des solutions. C'est là l'affaire des responsables politiques d'une société démocratique. Cependant, se peut-il que le gouvernement ait réalisé qu'il est nécessaire de changer de cap ? Après tout, le ministre de l'éducation John Patten a bien été remplacé par une ancienne enseignante et inspectrice de LEA, ancienne directrice d'un comité d'enseignement de LEA qui plus est. Elle s'est rendue sympathique au cœur des enseignants lorsqu'elle a dit en juillet dernier qu'elle les écouterait. J'espère qu'elle le fera. Je lui souhaite de réussir. Et quand elle écoutera, j'espère qu'elle entendra un message sonore et clair concernant les systèmes, l'organisation, les valeurs pédagogiques et non celles du marché. Nos élèves ne méritent rien de moins. Le marché n'est pas encore leur affaire.

\section{NOTES}

1. HMI (Her Majesty's Inspectorats of Schools) : corps d'inspection centrale distinct des inspecteurs ou conseillers pédagogiques régionaux; les HMI ont compétence sur l'ensemble du système éducatif, de l'enseignement préparatoire à l'enseignement supérieur (NdIR).

2. Office for Standards in Education

3. Grant maintained : école directement subventionnée par le ministère de l'éducation. 


\section{RÉSUMÉS}

Témoignage alerte et engagé d'un chef d'établissement bousculé par la réforme éducative anglaise: les changements introduits ces dernières années dans le fonctionnement des établissements sont générateurs d'une nouvelle culture professionnelle.

\section{INDEX}

Index géographique : Angleterre, Grande-Bretagne, Royaume-Uni

Mots-clés : chef d'établissement, établissement d'enseignement, personnel d'encadrement

\section{AUTEUR}

\section{BRYAN LOVE}

Principal du Bideford Community College, Devon, Angleterre

Bryan Love est chef d'un établissement secondaire situé à Bideford dans le Devon. Cet établissement désigné comme community college, est caractérisé par la diversité du public (d'une part, élèves âgés de 11 à 18 ans et, d'autre part, adultes en formation), et par la variété de l'enseignement dispensé : enseignement général, enseignement professionnel et activités de culture générale. 